\title{
COMPARING HEMOSTATIC PAIN AND ADHESION PREVENTION EFFECTS OF NASAL PACKING USED POST-SEPTUPLASTY VIZ MEDICATED GAUZE PACKING, AND MEROCEL PACKING
}

Vaibhav Kuchhal ${ }^{1}$, Prem Pal Singh ${ }^{2}$, Abhinav Srivastava ${ }^{3}$

\section{HOW TO CITE THIS ARTICLE:}

Vaibhav Kuchhal, Prem Pal Singh, Abhinav Srivastava. "Comparing hemostatic pain and adhesion prevention effects of nasal packing used post-septuplasty viz medicated gauze packing and merocel packing". Journal of Evolution of Medical and Dental Sciences 2013; Vol2, Issue 37, September 16; Page: 7160-7164.

ABSTRACT: The aim of this study was to compare the efficacy of a merocel technique with conventional nasal packing, in the classic septuplasty operation. The study is a prospective, randomized clinical trial. 60 patients underwent septuplasty for septal deviation and ensuing nasal obstruction. These patients were divided into two groups: conventional gauze packing and merocel packing. Randomization was performed using the two block randomization system. Patients who failed the regular follow-up were excluded, and the two groups were compared for postoperative bleeding, pain, hematoma, and adhesions. Patients were asked to record pain levels using a visual analogue scale. The authors found significant statistical differences between the two groups in the parameters studied, but significantly higher pain levels, Adhesions and withdrawal bleeding were noted in the patients in the gauze packing group. The final results confirmed that patients who underwent septuplasty with merocel packing had more smooth post-op recovery with less complication then conventional gauze packing.

KEY WORDS: Septuplasty, Conventional Gauze Packing, Merocel, Pain.

INTRODUCTION: Septuplasty is one of the common operations performed in ENT theatres. Among the known complications of septuplasty are postoperative hemorrhage, adhesion formation, and postoperative pain. Nasal packing postoperatively is done to prevent nasal bleeding and is thought to reduce adhesions, though effect of conventional nasal packing on adhesion formation or prevention is debatable.

The use of Merocel nasal packs can control bleeding in cases of epistaxis in about 91.5 percent of cases. It is hemostatic and can prevent adhesion formation. As is known if proper technique is followed during septuplasty, complications can be avoided including moderate to severe epistaxis. Many authors believe that nasal packing postoperatively is not needed if proper technique is followed and should thus be avoided to prevent significant morbidity associated with nasal packing. Some authors argue that packing should be reserved for cases where there is concern about persistent haemorrhage. Though these arguments carry enough weightage, packing of nasal cavities postoperatively is usually done in almost all parts of the world. Different types of nasal packing methods and materials are used to prevent many of the known complications of septuplasty. This study is undertaken to compare the advantages and disadvantages of two types of packings viz, medicated gauze packing and merocel packing. 


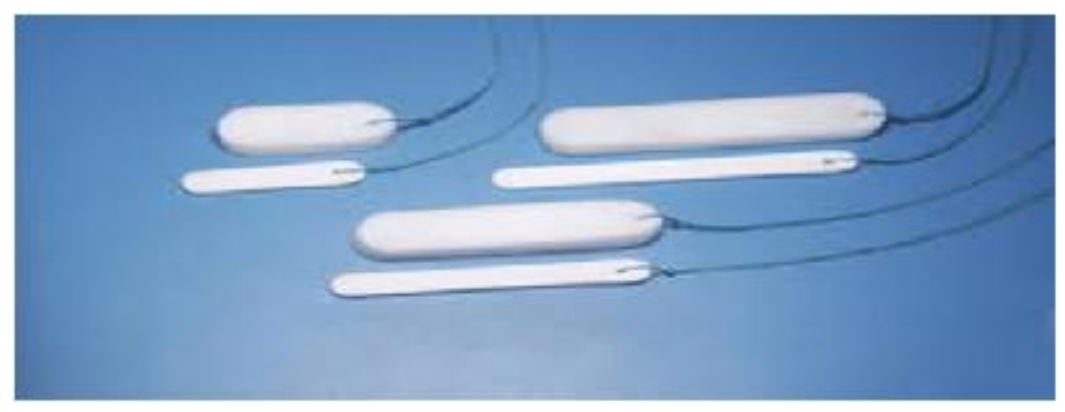

METHODS: A total of 60 patients were randomly assigned in two Groups A and B.

Postoperative patients in Group A were packed with Classical Medicated Gauze packing. In Group B, patients were simply packed with merocel.

All the septoplasties were done under local anesthesia so that in the immediate postop period patients can maintain sitting posture which to a certain extent ensures that if patient bleeds, it does so anteriorly from where it can be measured or quantified.

In all the two groups, patients were provided with cotton balls to clean the blood tinged soakage and keep these cotton balls preserved for quantification. Each cotton ball was roughly the size of an average sized walnut. Patients who needed repacking were left out from the study because in them bleeding could not be quantified according to the protocol of our study.

Packing was removed after 72 hours and for these 72 hours patients were put on i.v. antibiotics and systemic decongestants. Postoperatively patients were followed at following week/s for adhesion formation and bleeding:

- 1 week

- 4 weeks

\section{Inclusion Criteria:}

1. Patients undergoing septuplasty in the age range of 18 to 60 years.

2. Patients of both sexes were included in the study.

\section{Exclusion Criteria:}

1. Elderly patients above 60 years and children under 18 years of age.

2. Females presently having menstrual cycle.

3. Patients with revision Septoplasties.

4. Patients with systemic disorders like Collagen disorders, diabetes where poor healing is suspected.

5. Patients with bleeding or coagulation disorders.

6. Patients lost to follow up.

7. Patients who needed repacking post septuplasty

RESULTS: In the immediate postoperative period, patients were intimately observed for the bleeding. The numbers of cotton balls used were counted. Care was also taken to quantify the amount of soakage that each cotton ball had and accordingly the number of cotton balls used was adjusted by addition or subtraction from the number of cotton balls actually used. 
Following table lists the average no. of cotton balls used in two groups on day $1 \& 2$.

\begin{tabular}{|c|c|c|}
\hline Day & Group A & Group B \\
\hline Day 1 & 10 & 15 \\
\hline Day 2 & 5 & 7 \\
\hline \multicolumn{3}{|c|}{ Table-1: Number of cotton balls used } \\
\hline
\end{tabular}

As is evident, on day 1, conventional gauze packing was apparently better hemostatic than merocel packing. Applying Chi square test between conventional packing and merocel packing as under

- Mean no. of cotton balls used in conventional ANP (Observed), $0=10$

- Mean no. of cotton balls used in merocel

Packing (Expected), e = 15

- $\chi^{2}=(\mathrm{o}-\mathrm{e}) 2 / \mathrm{e}$

$=(15-10) 2 / 10$

$=2.5$

- Here degrees of freedom, $n=1$

- Computing ' $p$ ' value from Chi square distribution, $p<0.20$ As is evident, the difference is not statistically significant even though conventional ANP appeared to be better in controlling bleeding from nose.

\begin{tabular}{|c|c|}
\hline Group A & Group B \\
\hline 10 patient & 0 patient \\
\hline $16 \%$ & $0 \%$ \\
\hline $\begin{array}{c}\text { Table-2: At 4 weeks postoperatively, following } \\
\text { no. (percentage) of patients developed adhesions }\end{array}$ \\
\hline
\end{tabular}

Application of $\chi^{2}$ test between conventional

ANP and merocel packing:

- Number of patients developing

adhesions, $=0$ (Merocel packing)

- Number of patients developing

adhesions, $\mathrm{e}=10$ (Conv. packing)

- $\chi^{2}=(\mathrm{o}-\mathrm{e}) 2 / \mathrm{e}$

$=(0-10) 2 / 10$

$=10$

Here $\mathrm{p}$ value comes out to be, 0.002 (degree of freedom is 1). Thus the difference is statistically significant and the reason why merocel packing should be done in all cases following septuplasty.

For pain management all patients were put on b.d dosage of $500 \mathrm{mg}$ paracetamol tablets on first two days and then on S.O.S basis thereafter. Pain assessment was done by calculating the no. of tablets consumed in the first postoperative week.

Following table depicts the same 


\section{CLINICAL STUDY}

\begin{tabular}{|c|c|}
\hline Group A & Group B \\
\hline 15 Tablets & 8 Tablets \\
\hline \multicolumn{2}{|c|}{ Table-3: Pain management outcome } \\
\hline
\end{tabular}

Thus minimal pain was seen with merocel packing, which is also the reason why postoperatively patient should be packed with merocel.

CONCLUSION: Hemostatic effects were best observed with medicated gauze packing, though the difference was not statistically significant. Adhesion formation which is one of the most important determinants of success of septuplasty is best prevented by merocel packing. If good expertise is observed during septuplasty with proper sterilization technique, post septuplasty hemorrhage is quite uncommon and adhesion prevention becomes the single most important factor. Merocel packing should thus be carried out in almost all cases. In cases where postoperative bleeding is suspected, classical medicated gauze packing should be done. The morbidity associated with postoperative pain was minimal with merocel packing.

DISCUSSION: This study revealed that adhesion formation which happens to be one of the most important factors in preventing the successful outcome of surgery is best prevented.

This besides offering the freedom from adhesions also offers freedom from secondary hemorrhage on account of adhesions and avoids morbid pain that is associated with the procedure of releasing adhesions. Hemostatic effects are best observed with conventional medicated gauze packing although the results were not statistically significant on Chi square test. If septuplasty is done by a skillful surgeon using proper infiltration technique (1:100000 adrenaline) and proper sterilization technique, the risk of moderate or severe epistaxis is remote and adhesion prevention becomes the most important factor to be dealt with. It is thus advised to use merocel packing in all septoplasties, if minimal bleeding is suspected postoperatively. But if the suspicion of moderate to severe epistaxis is high as with significantly traumatic surgeries, malnourished children, hypertensives on erratic treatment etc. conventional ANP is advisable.

Additionally, postoperative pain due to nasal packing is of lower intensity by merocel packing. It is thus advised to use merocel packing in all patients undergoing septuplasty as well as duration with the use of merocel packing when compared with other types of packing. Thus considering the advantages of merocel packing in preventing adhesions, and the significantly lower postoperative pain, and avoidance of revision procedures to release adhesions; it is advisable to use this packing in all patients undergoing septuplasty.

\section{References:}

1. White JAM Murray. Intranasal adhesion formation following surgery for chronic nasal obstruction. Article first published online: 2 AUG 2007; DOI: 10.1111/ j.13652273.1988.tb00754.x.

2. Pringle MB, Beasley $P$ and BrightwellAP. The use of merocel nasal packs in the treatment of epistaxis. J LaryngolOtol 1996; 110: 543-546.

3. Mcintosh David, Cowin Allison, Adams Damian, Wormald Peter-John. The Effect of an Expandable Polyvinyl Acetate (Merocel) Pack on the healing of the nasal mucosa of sheep. American Journal of Rhinology, 19(6): Publication date: 2005-11-01. 
4. Bloom JD, Kaplan SE, Bleier BS, Goldstein SA. Septuplasty complications: avoidance and management. OtolaryngolClin North Am. 2009; 42(3): 463-81.

5. Nunezi DA, Martin FW, An evaluation of post-operative packing in nasal septal surgery. Clinical Otolaryngology \& Allied Sciences 1991; 16(6): 549-550.

6. Mohammad Sohail Awan, Iqbal Moghira; Nasal packing after septuplasty: A randomized comparison of packing versus no packing in 88 patients. Ear, Nose \& Throat Journal 2008; 87: 624-627.

7. Marcelle Von Schoenberga, Philip Robinsona and Rowena Ryana. Nasal packing after routine nasal surgery-is it justified? J LaryngolOtol 1993; 107: 902-905.

8. James A. Cook, Nicholas J Murrant, Katel. Evans, Richard J Lavelle. Intranasal splints and their effects on intranasal adhesions and septal stability. Clinical Otolaryngology \& Allied Sciences 1992; 17(1): 24-27.

\section{AUTHORS:}

1. Vaibhav Kuchhal

2. Prem Pal Singh

3. Abhinav Srivastava

\section{PARTICULARS OF CONTRIBUTORS:}

1. Associate Professor and Head, Department of ENT, GMC, Haldwani.

2. Junior Resident, Department of ENT, GMC, Haldwani.

3. Senior Resident, Department of ENT, GMC, Haldwani.

\section{NAME ADDRESS EMAIL ID OF THE} CORRESPONDING AUTHOR:

Dr. Vaibhav Kuchhal,

Associate Professor and Head, Department of ENT,

GMC, Haldwani - 263139.

Email-vaibhavgarima@gmail.com

Date of Submission: 05/09/2013. Date of Peer Review: 06/09/2013. Date of Acceptance: 10/09/2013. Date of Publishing: 16/09/2013 